far and how long to do. The blood analysis has not been described until now, as possible predictor of survival in these patients. Aim: To analyze $\mathrm{pH}$, lactate, bases excess (BE) and pCO2 as possible predictive factors of survival in patients who suffer TCA.

Method Observational Study of patients suffered from TCA in 2016, 2017 and 2018 assisted by our EMS. Collection data from medical records and databases of hospital follow-ups. Data processing and data analysis: quantitative variables are described by central and dispersion measures and qualitative variables by frequency distribution. COR as survival analysis. Excel and SPSS v. 20.0.

Results We analyzed 112 TCA in which CPR is performed and recovered spontaneous circulation 49 (43.75\%). After 7 days, $7.14 \%$ survived. At the arrival EMS, mean $\mathrm{pH}$ was 7.14 (SD 0.15), pCO2 66.57 (ED 20.61), BE -6.09 (ED 6.23) and Lactate 6.51 (ED 3.82). Lactate and PCO2 showed significant relationship in analysis of survival curve after 7 days $(\mathrm{p}<0,05)$.

Conclusion The survival in TCA was $7.14 \%$ of patients after 7 days. - Lactate and PCO2 are related to survival at 7 days in our series. - It's necessary more robust studies that can define the factors related to the survival in TCA, but it seems that the biological clock can be one of them.

Conflict of interest There is no conflict of interest.

Funding There is no funding.

\section{TRAUMATIC CARDIAC ARREST: IS SURVIVAL POSSIBLE?}

C Camacho*, G Mancho, E Pastor, MJ Garcia-Ochoa, E Corral. SAMUR Protección Civil, Madrid, Spain

\subsection{6/bmjopen-2019-EMS. 18}

Background Survival in traumatic PCR has been considered almost impossible, so it was even question whether it was indicated to perform CPR. Nowadays, several studies publish variable survival rates but they are a reality.

Aim To analyze the survival of patients suffering from traumatic CRP treated by an emergency service.

Method Observational study of patients suffered from traumatic cardiac arrest in 2016, 2017 and 2018 assisted by our EMS. Collection data from medical records and databases of hospital follow-ups. Scope: City of Madrid. Data processing and data analysis: quantitative variables are described by central and dispersion measures and qualitative variables by frequent distribution. Excel and SPSS v.20.0.

Results 303 cardiac arrest of traumatic origin were selected, of which CPR is performed in $155(51.2 \%)$. Of these, CPR was abandoned for futility in $43(27.74 \%)$. Rest of patient were 112 (with complete CPR), 49 (43.75\%) recovered the spontaneous circulation. After 6 hours, 33 patients were alive (29.46\%). After 24 hours $16.96 \%$ and after 7 days $7.14 \%$.

Conclusion Survival in traumatic cardiac arrest is possible, $7.14 \%$ of patients are alive after 7 days. - Our survival rates are comparable to those of other series published in the scientific journals. - It's possible that survival will improve in the next years due to the new management of TCA.

Conflict of interest There is no conflict of interest.

Funding There is no funding.

\section{LIVESTREAMING FROM SMARTPHONES TO THE} DISPATCH CENTER IN REAL EMERGENCY CALLS

${ }^{1,2} \mathrm{G}$ Linderoth*, ${ }^{3} \mathrm{D}$ Østergaard, ${ }^{1} \mathrm{M}$ Fjordholt, ${ }^{1} \mathrm{~F}$ Folke, ${ }^{1} \mathrm{~F}$ Lippert. ${ }^{1}$ Emergency Medical Services Copenhagen, University of Copenhagen, Copenhagen, Denmark; ${ }^{2}$ Department of Anaesthesia and Intensive Care, Bispebjerg and Frederiksberg Hospital, University of Copenhagen, Copenhagen, Denmark; ${ }^{3}$ Copenhagen Academy for Medical Education and Simulation, University of Copenhagen, Herlev, Denmark

\subsection{6/bmjopen-2019-EMS.19}

Background Currently, the emergency medical dispatchers rely solely on the spoken word when assessing and triaging emergency calls. We aimed to explore if livestreaming by bystanders could be useful during emergency calls.

Method In a 3 months period, livestreaming could be added to the emergency call at the Emergency Medical Services, Copenhagen. Nine medical dispatchers were trained to instruct laypersons to ad livestreaming at a 1 day simulation-based course before the period. For livestreaming caller received a text message with a link. When activating the link, the smartphone camera opened, allowing video to be streamed encrypted to the dispatcher. GoodSAM provided the technical solution.

Results In 68 cases, the caller had a smartphone and the dispatcher suggested livestreaming which succeeded in 33 cases. Reasons for no livestreaming were refusal from bystander $(n=6)$ or patient $(n=2)$, text message not received before ambulance arrival $(n=17)$, and technical issues or caller skills $(n=11)$. The dispatchers found the live video recording useful in all cases. In 8 cases (24\%) the patient was considered more critical ill when livestreaming was added, whereas in $8(24 \%)$ cases the patient was considered less sick. Change in ambulance priority response was done in 3 cases after the dispatchers had video. Among 25 callers interviewed 22 experienced livestreaming as an advantage.

Conclusion Adding video livestreaming to the emergency call seems useful for the medical dispatchers to improve patient assessment and to provide the appropriate emergency response. The callers found livestreaming an advantage. Technical issues/experiences, however, need to be improved.

Conflict of interest The authors declare that they have no competing interests.

Funding Emergency Medical Services Copenhagen: has received unrestricted research grants from the Laerdal Foundation for acute medicine and from the Danish foundation TrygFonden. CAMES has received unrestricted research grants from the Laerdal Foundation.

\section{IMPACT OF PHYSICIAN-STAFFED HELICOPTERS ON PATIENT OUTCOMES: A SYSTEMATIC REVIEW}

${ }^{1} \mathrm{C}$ Draegert*, ${ }^{2} \mathrm{~B}$ Risgaard, ${ }^{2} \mathrm{JS}$ Baekgaard, ${ }^{2} \mathrm{~J}$ Steinmetz, ${ }^{2} \mathrm{LS}$ Rasmussen. ${ }^{1}$ University of Copenhagen, Denmark; ${ }^{2}$ Department of Anesthesia, Center of Head and Orthopedics, Rigshospitalet, Denmark

\subsection{6/bmjopen-2019-EMS.20}

Background Management of pre-hospital patients remains a challenge. In developed countries a physician-staffed helicopter emergency medical service (PS-HEMS) is used in addition to ground emergency medical service (GEMS), but the effect is debated. This systematic review aimed to evaluate the effect 